'Departamento de Obstetricia y Ginecología, Facultad de Medicina, Universidad de Concepción. Concepción, Chile. ²Departamento de Educación Médica, Facultad de Medicina, Universidad de Concepción. Concepción, Chile.

${ }^{3}$ Facultad de Medicina, Universidad de Concepción. Concepción, Chile.

${ }^{a}$ Médico Cirujano especialista en Obstetricia y Ginecología, Magister en Educación para Ciencias de la Salud.

bsicóloga, Magíster en

Ciencias de la Educación, mención Didáctica e Innovación Pedagógica.

'Estudiantes de pregrado de medicina.

Trabajo no recibió financiamiento. Los autores declaran no tener conflictos de interés.

Recibido el 29 de agosto de 2018, aceptado el 15 de marzo de 2019.

Correspondencia a: Henry Castro Arias Chacabuco esquina Janequeo, Facultad de Medicina Universidad de Concepción, Concepción, Chile. hncastro@gmail.com henrycastro@udec.cl

\section{Determinación de pensamiento creativo en estudiantes de medicina de una universidad chilena}

\author{
HENRY CASTRO A. ${ }^{1, \mathrm{a}}$, JAVIERA ORTEGA B. ${ }^{2, \mathrm{~b}}$, \\ JAVIER VILLARROEL L. ${ }^{3, c}$, CARLA CONTRERAS S. ${ }^{3, c}$
}

\section{Assessment of creative thinking among medical students}

Background: Creativity is a highly valued quality in different fields. Despite this, it is rarely included in the curricula of medical careers. Aim: To assess creative thinking among undergraduate medical students. Material and Methods: Sixty-seven students aged 18 to 31 years (45\% women) from the first (16), third (26) and seventh year (25) of medical school answered a sociodemographic questionnaire and the Torrance Test of Creative Thinking (TTCT). Results: Median creativity index according to age was in percentile 52 (range 2-99). Percentiles for fluency, originality, elaboration, abstraction of titles and resistance for premature closure were 31, 32, 79, 53 and 17 respectively. There were no significant differences between students of different levels $(p=0.73)$. Conclusions: In this group of students, there is a high performance in elaboration and a low score in Resistance to premature closure.

(Rev Med Chile 2019; 147: 372-377)

Key words: Creativity; Curriculum; Learning; Students, Medical; Thinking.
L a creatividad es una cualidad muy valorada. En Estados Unidos de Norteamérica se considera como una de las competencias más deseables en postulaciones a escuelas de postgra$\mathrm{do}^{1}$. Asimismo, ha sido declarada como el recurso económico más importante del siglo $\mathrm{XXI}^{2}$ lo cual se refleja en el incremento explosivo de empleos asociados desde $1980^{3}$. Definir creatividad ha sido siempre un desafío ${ }^{4}$. Una de las definiciones más aceptadas, propuesta por Runco, afirma que la creatividad debe tener dos características principales: originalidad y utilidad ${ }^{4}$. La originalidad hace referencia a lo nuevo, o bien lo nunca visto, $y$ utilidad significa que toda creación debe ser útil en algún aspecto. Runco remarca que la originalidad por sí sola no aporta a la comunidad, perdiendo el sentido funcional de la creatividad ${ }^{4-6}$. En la cultura oriental, sin embargo, el individualismo o la originalidad no pareciera ser un valor importante. Per- seguir el bien colectivo o utilidad, la espiritualidad, la estética es de mayor valor ${ }^{7}$. También utilidad es cuestionada, aunque Bethune en 1839, refirie que el esfuerzo del intelecto debe buscar hacer al hombre más sabio, mejor y más feliz ${ }^{4}$.

El estudio de la creatividad se divide en cuatro componentes: personalidad, proceso creativo, producto y ambiente ${ }^{8,9}$. La medición del pensamiento divergente es una forma de evaluar el proceso creativo ${ }^{10,11}$. Pensamiento divergente es la habilidad para explorar posibilidades, asociaciones e interpretaciones poco convencionales, con el fin de producir un amplio rango de ideas ante un problema $^{12}$. En tanto, pensamiento convergente es la habilidad de combinar o analizar varias ideas y llegar a una solución posible ${ }^{12}$. Existen diversos test para medir pensamiento divergente, pero el más utilizado es el Test de Torrance, por su evidencia de validez y confiabilidad ${ }^{13}$. Desarrollado en 1968 y 
actualizado acorde a las nuevas cohortes y cambios del entorno, su objetivo es evaluar características del pensamiento, como fluidez, originalidad, resistencia al cierre, elaboración y abstracción de títulos, además de fortalezas creativas ${ }^{14,15}$.

En Medicina poco o nada existe sobre estudios de la creatividad. A pesar de ser una cualidad altamente valorada por estudiantes, pareciera no ser así cuando se analizan las representaciones de pacientes, quienes ven a los médicos como sujetos competentes o profesionales, pero en ningún caso como creativos ${ }^{16}$.

Específicamente en educación médica, la creatividad no ha sido considerada como un eje central de la práctica docente. Considerando que ejercer una disciplina científica que tiene como esencia su proceso de actualización, se debiese considerar a la creatividad un eje reflexivo primario. Por otro lado, se ha observado que cuando se otorgan las condiciones necesarias, los alumnos pueden desarrollar pensamiento divergente. Entonces, ¿por qué durante la formación, parecieran no encontrar cabida las soluciones creativas? Los distintos currículos tienden a basarse en adquisición de contenidos, su aplicación y evaluación a través de una secuencia predeterminada. Esto deja muy poco espacio a la creatividad ${ }^{17}$.

Los currículos de distintas escuelas han visto la necesidad de incorporar la creatividad, sin embargo, los reportes son anecdóticos de asignaturas asociadas al arte. Aun así, esta implementación innovadora ha demostrado traer consecuencias positivas en los estudiantes ${ }^{18}$.

El proceso creativo impulsa el aprendizaje incitando a los estudiantes a buscar soluciones y hacer reflexiones entre los hechos y experiencias reales, haciendo que uno de los beneficios sea el proceso reflexivo ${ }^{18}$. El aprendizaje transformador que implica cambios de actitud y perspectiva, puede ser reforzado por el proceso creativo, porque implica un compromiso completo del individuo en el proyecto, no solo de manera intelectual, sino también emocional. Otros beneficios son la generación de nuevos conocimientos, el fomento del desarrollo profesional y la capacidad de transformar barreras en oportunidades de aprendizaje. Los alumnos refieren que aquellos programas que incluyen la creatividad, les provocan una sensación de crecimiento personal y desarrollo, además de una sensación de comunidad, mejorando sus habilidades de trabajo en equipo y colaboración, destacando la conciencia de la existencia de valores humanísticos en medicina ${ }^{19}$.

Poco conocemos acerca del desarrollo de la creatividad en medicina y del potencial de nuestros alumnos. No existen estudios publicados que diagnostiquen pensamiento creativo en estudiantes de medicina en Chile. Un punto importante cabe mencionar: tal como Torrance lo expuso, un buen resultado no asegura que el individuo va a comportarse de manera creativa ${ }^{20}$, pues depende de otros factores. Sin embargo, el test tiene un valor predictivo para señalar quienes tendrán más posibilidades de desarrollar la creatividad en algún momento de su vida, lo cual fue reafirmado en estudios de seguimiento a los 40 y 50 años ${ }^{15,21}$.

Este estudio se enmarca en el inicio de una línea de investigación relacionada con el desarrollo de la creatividad en medicina y tiene como objetivo establecer un diagnóstico de pensamiento creativo a través de la medición del pensamiento divergente entre alumnos de pregrado de medicina de distintos niveles.

\section{Materiales y Método}

Se utilizó una metodología cuantitativa con diseño no experimental, de corte transversal y alcance correlacional. Los participantes fueron estudiantes de primero, tercero y séptimo año de la carrera de medicina de la Universidad de Concepción. Criterios de inclusión fueron ser alumno regular de la carrera y que hubieran cursado la mayoría de asignaturas del nivel correspondiente. Criterios de exclusión fueron tener alguna patología de salud mental o neurológica y tener menos de 18 años. El muestreo utilizado fue no probabilístico de carácter incidental ${ }^{22}$, lo que permitió alcanzar una muestra total de 67 estudiantes. El proceso de aplicación de los instrumentos, requería a los participantes una hora.

Como instrumento de recolección de datos, se utilizó el Test de Pensamiento Creativo de Torrance (TPCT) en su forma A de figuras validada al español y autorizada por la Scholastic Testing Service (SST) Inc $\odot$ y un cuestionario sociodemográfico validado por juicio de expertos. El TPCT consta de tres partes basadas en la realización de dibujos en base a distintos estímulos en un tiempo determinado. La primera parte es acerca de construcción de dibujos, la segunda 
completación de dibujos y la tercera acerca de figuras repetidas. Cinco subescalas son medidas en este test: 1) Fluencia, que refiere al número de ideas relevantes; 2) Originalidad, que refiere al número estadístico de respuestas infrecuentes; 3) Elaboración, es el número de ideas adicionadas a un concepto inicial; 4) Abstracción de títulos, mide la capacidad del sujeto de elaborar un título abstracto que represente la idea dibujada, y 5) Resistencia al cierre prematuro, refleja el grado de apertura psicológica necesario para procesar una variedad de información. Además, el test evalúa 13 criterios llamados "fortalezas creativas". La suma de los puntajes de las 5 variables principales se refleja en el Puntaje Estándar Promedio generando el Índice de Creatividad. Tanto el puntaje estándar promedio como el Índice de Creatividad son medidos en percentiles de acuerdo a edad y curso. La recomendación de aplicación en adultos es homologar al valor más alto de edad y de curso. La confiabilidad del índice de creatividad para curso es de 0,89 y para edad de 0,90. En tanto, la confiabilidad por dominio, es de 0,99 para fluencia, 0,95 para elaboración, 0,96 para resistencia al cierre prematuro, 0,97 para originalidad y 0,96 para abstracción de títulos ${ }^{13}$.

La aplicación del TPCT fue en grupos del mismo curso, según normas establecidas por STS Inc@ en el período 2016-2017. Así mismo, la evaluación de cada test fue realizado por un autor según normas establecidas por la STS, en base al Streamlined Scoring Guide for Figural Forms A and $B$, SST Inc@. Posteriormente, la determinación del Puntaje Promedio Estándar, del Índice de Creatividad y de los percentiles correspondientes, fueron establecidos en base a los lineamientos de Norms-Technical Manual Figural (Streamlined) Forms A\&B, SST Inc $($.

Como plan de análisis se utilizó estadística descriptiva con la finalidad de identificar los niveles de pensamiento creativo y aspectos socio-demográficos de la muestra y los puntajes que obtuvieron en el TPCT. Se determinó los niveles de pensamiento creativo en alumnos de medicina durante distintas etapas del programa de pregrado, y se establecieron tres grupos según los resultados. Para ello, se sumaron los puntajes estandarizados totales de las variables y se recodificó en una nueva variable categórica o cualitativa, denominada creatcat ${ }^{22}$. Los grupos quedaron conformados por aquellos alumnos con puntaje menor al percentil 25 , un segundo grupo con alumnos que obtuvieron un percentil entre 25 y 75 y un tercer grupo con alumnos que obtuvieron un percentil sobre 75 . Finalmente, los alumnos fueron separados según el nivel o curso al cual pertenecían. Se realizó un análisis bivariado utilizando la prueba $\chi^{2}$ con significancia $\mathrm{p}<0,05$. Se utilizó software SPSS 21.0 para el análisis estadístico.

Estudio aprobado por Comité de Ética de la Facultad de Medicina de la Universidad de Concepción. Todos los participantes firmaron el consentimiento informado previo a la aplicación de la batería de test y tuvieron la oportunidad de retirarse del estudio en cualquier momento.

\section{Resultados}

Participaron 67 estudiantes. Edad media de 22,34 años (18 a 31 años). En cuanto al sexo, 37 participantes $(55,2 \%)$ son hombres y 30 participantes $(44,8 \%)$ son mujeres.

La distribución por nivel o curso es la siguiente: $23,9 \%(\mathrm{n}=16)$ corresponde a primer año, 38,8\% $(\mathrm{n}=26)$ a tercer año y $37,3 \%(\mathrm{n}=25)$ a séptimo año.

Según sus estudios de enseñanza media, el $9,1 \%(\mathrm{n}=6)$ de los participantes proviene de un establecimiento municipalizado, $53 \%(\mathrm{n}=35)$ de un establecimiento particular subvencionado y el $37,9 \%(n=25)$ de un establecimiento particular pagado.

El 100\% $(n=67)$ declara su estado civil como soltero/a, en tanto $3(4,5 \%)$ de los participantes tienen hijos. Sesenta y siete participantes correspondientes a $100 \%$ de la muestra desarrollaron el TPCT. Los resultados por variable se detallan en la Tabla 1.

Se observaron diferencias porcentuales entre los tres niveles formativos. Respecto a los alumnos de primer año, el $25 \%(\mathrm{n}=4)$ se encuentra en el grupo menor a percentil $25,31,25 \%(n=5)$ se encuentra en el grupo p25-75 y 43,75\% $(n=7)$ se encuentra en el grupo mayor a p75. Entre los alumnos de tercer año, el 34,61\% $(\mathrm{n}=9)$ se encuentra en el grupo menor a p25, 11,53\% $(n=3)$ se encuentra en el grupo p25-75 y 53,8\% $(n=14)$ se encuentra en el grupo mayor a p75. Finalmente, entre los alumnos de séptimo año, 36\% $(\mathrm{n}=9)$ se encuentra en el grupo menor a p25, 8\% $(n=2)$ se encuentra en el grupo p $25-75$ y 56\% $(n=14)$ se 
Tabla 1. Resultados descriptivos obtenidos en pensamiento creativo con el Test De Torrance

\begin{tabular}{|lcccc|}
\hline Variable & n & Mínimo & Máximo & Media \\
\hline Fluencia (edad) & 67 & 2 & 82 & 31,33 \\
\hline Originalidad (edad) & 67 & 1 & 99 & 32,21 \\
\hline Elaboración (edad) & 67 & 4 & 99 & 79,85 \\
\hline Abstracción de títulos (edad) & 67 & 3 & 97 & 53,54 \\
\hline Resistencia al cierre prematuro (edad) & 67 & 1 & 52 & 17,66 \\
Puntaje Índice de Creatividad (edad) & 67 & 57 & 150 & 110,13 \\
\hline Percentil Índice de Creatividad (edad) & 67 & 2 & 99 & 52,94 \\
\hline Puntaje total Checklist de fortalezas creativas & 67 & 6 & 24 & 15,21 \\
\hline Percentil de Puntuación media estándar (edad) & 67 & 1 & 99 & 43,12 \\
\hline
\end{tabular}

encuentra en el grupo mayor a p75. No se observaron asociaciones estadísticamente significativas entre los niveles de pensamiento creativo y el nivel que cursan los participantes ( $\mathrm{p}=0,73$ ).

\section{Discusión}

Los resultados arrojados demuestran que el grupo de alumnos se distribuye en una media de percentil promedio típico en comparación con los estándares de adultos de Estados Unidos. Sin embargo, destaca el grupo de alumnos sobre el percentil 75 , siendo el más numeroso en los tres niveles. Es importante entender que el TPCT, si bien es una medición de pensamiento divergente, también es un predictor de logros creativos ${ }^{15}$, por lo tanto, estos resultados implican que un grupo importante de la cohorte eventualmente podría desarrollar creatividad tanto en el ámbito personal como público. Esto es dependiente de múltiples factores como el ambiente, oportunidades y rasgos de personalidad ${ }^{21}$, ya que el test por si solo no asegura el desarrollo de la creatividad.

$\mathrm{Al}$ analizar las subescalas del test, destaca la alta puntuación en Elaboración y la baja puntuación en Fluencia, lo cual ha sido descrito como una cualidad de los adultos creativos eminentes. Estos individuos, habitualmente parten en su juventud o niñez con altos puntajes en fluencia, pero a medida que envejecen, el número de ideas disminuye a costa de incrementar la elaboración; esto significa, que los adultos prefieren desarrollar menos ideas, pero más acabadas ${ }^{23}$. Otro de los aspectos que destacan en los resultados es el bajo puntaje obtenido en Resistencia al cierre prematuro. Esta variable presenta una fuerte relación positiva con inteligencia, $y$ ante el incremento de la inteligencia en los últimos años según estudios de medición de $\mathrm{IQ}^{24}$, se esperaba que esta también aumentara. Sin embargo, se ha visto en otros estudios una disminución de este indicador, lo cual no significa que los individuos no sean inteligentes, si no que indica que los jóvenes están creciendo con la mente más cerrada, menos curiosos y menos abiertos a nuevas experiencias ${ }^{23}$.

Por último, las Fortalezas creativas también han mostrado una reducción en los puntajes a través de los años, lo cual ha sido atribuido a características de los niños, que se han vuelto menos energéticos, menos emocionalmente expresivos, menos imaginativos ${ }^{23}$. En esta cohorte, contrario a esta tendencia, los resultados fueron muy buenos en la mayoría de las fortalezas, sobre todo las emocionales, pero si presentan bajos puntajes en humor y síntesis, lo cual podría atribuirse a características emocionales encontradas en el estudiante de medicina como empatía, que es base del profesionalismo, buen cuidado del paciente y habilidades de comunicación ${ }^{25}$.

Respecto a diferencias que podrían haber existido entre distintos niveles, no se demostró diferencias significativas. Esto también es corroborado por estudios previos ${ }^{23}$ en que los adultos tienden a comportarse de manera estable, a diferencia de los niños, que presentan cambios marcados durante los años de la educación básica. Con este resultado, podría inferirse que la escuela de medi- 
cina no es un factor que deteriore los puntajes de pensamiento creativo, sin embargo, para probar esta afirmación, se debe seguir una cohorte en el tiempo y estudiar las diferencias en el mismo grupo de alumnos.

El estudio de la creatividad y específicamente del pensamiento creativo no está estandarizado, y esta es una limitación de este estudio, ya que solo entrega un punto de medición. Es importante entonces entender, que estos resultados, no son un reflejo exacto de creatividad, si no, un aspecto. Por otra parte, tanto el tamaño muestral como el plan de captación de voluntarios pudiesen influir en los resultados. A pesar de esto, la principal fortaleza de este estudio es que entrega un diagnóstico acerca de una población no estudiada en Chile y además, considerando que la Educación Superior ha enfatizado la necesidad de incorporar diversas competencias profesionales en la formación de pregrado $^{26,27}$, la creatividad emerge como una habilidad que también tiene un valor importante.

Finalmente, podemos concluir que los estudiantes de medicina presentan niveles de pensamiento creativo similar a cualquier adulto. A pesar de no existir asociaciones estadísticamente significativas entre ellos, se evidenció diferencias porcentuales en los diversos niveles formativos. La pregunta que nos lleva lo anterior, es pensar si es necesario profundizar, como también poner en discusión si en el contexto socioeconómico y político actual se hace necesario promover el pensamiento creativo en alumnos de medicina. Esto llevaría a las escuelas de medicina al menos a plantear si la creatividad es una característica o competencia necesaria de sus egresados. Y si fuera así, deberemos discutir cómo lograríamos integrar estrategias de enseñanza que promuevan pensamiento creativo en la formación.

\section{Referencias}

1. Enright M. Toward a Description of Successful Graduate Students, 1989.(GRE Board Professional Rep. No. 8909, GRE Board Research Rep. 85-17R). Princeton, NJ: Educational Testing service.

2. Florida R. The rise of the creative class. 2002. New York: Basic Books.

3. Florida R. The flight of the creative class. Liberal Education 2006; 22-9.

4. Runco M, Jaeger G. The standard definition of creativi- ty. Creativity Research Journal 2012; 24 (1): 92-6.

5. Kaufman J. Beyond Big and little: The four C Model of creativity. Review of General Psychology 2009; 13 (1): $1-12$.

6. Brunner JS. The conditions of creativity. In Gruber H, Terrell G, Wertheimer M. Contemporany approaches to creative thinking. New York: Atherton; 1962. p. 1-30.

7. Kharkhurin A. Creativity. 4 in 1: four-criterion construct of creativity. Creativity research journal 2014; 26 (3): 338-52.

8. Zeng L. Creativity in ergonomic design: a supplemental value-adding source for product and service development. Human factors and ergonomic society 2010; 52 (4): 503-25.

9. Rhodes M. An Analysis of Creativity. The Phi Delta Kappan 1961; 42 (7): 305-10.

10. Sternberg RJ. The assessment of creativity: an investment-based approach. Creativity Research Journal 2012; 24 (1): 3-12.

11. Boada-Grau J, Sánchez-García J, Prizmic-Kuzmica A. Adaptación de la escala Creative Environment Perceptions al español. Univ Psychol 2014; 13 (4): 1357-66.

12. Zeng L. Can traditional divergent thinking tests be trusted in measuring and pre-dicting real world creativity?. Creativity Research Journal 2011; 23 (1): 24-37.

13. Torrance E. Paul. Torrance Tests of Creative Thinking. Norms-Technical Manual. Figural (Streamlined) forms A \& B $\odot$ 1998, 2008 Scholastic Testing Service, Inc.

14. Torrance E. Prediction of adult creative achievement among high school seniors. Gifted Child Quarterly 1969; 13 (4): 223-9.

15. Runco M. Torrance Test of Creative Thinking as predictors of personal and pub-lic achievement: a fifty-year follow up. Creativity Research Journal 2010; 22 (4): 3618.

16. Lippell S. Creativity and medical education. Medical Education 2002; 36: 519-21.

17. Shaywitz DA. Preserving Creativity in Medicine. PLoS Med 2004; 1 (3): e34.

18. Jones EK. Creative art and medical student development: a qualitative study. Medical Education 2017; 51 (2): 174-83.

19. Thompson T. Compulsory creativity: rationales, recipes, and results in the placement of mandatory creative endeavor in a medical undergraduate curriculum. Medical Education Online 2010; 15: 5394.

20. Torrance E. Predictive validity of the Torrance Test of Creative Thinking. The Journal of creative Behavior 1972; 6 (4): 236-52.

21. Cramond B. A Report on the 40-year follow up of the Torrance Test of Creative Thinking: Alive and Well in 
the New Millennium. Gifted Child Quaterly 2005; 49 (4): 283-91.

22. Pardo A, Ruiz MA, San Martín R. Análisis de datos en ciencias sociales y de la salud I. Madrid: Síntesis. 2009.

23. Hee Kim K. Creativity crisis: the decrease in creative thinking scores on the Tor-rance Tests of Creative Thinking. Creativity Research Journal 2011; 23 (4): 285-95.

24. Pietschnig J. One Century of global IQ gains: A formal meta Analysis of the Flynn effect (1909-2013). Perspectives on Psychological Science 2015; 10 (3): 282-306.

25. Arora S. Emotional intelligence in medicine: a systema- tic review through the con-text of the ACGME competencies. Medical Education 2010; 44: 749-64.

26. Anvik T, Gude T, Grimstad H, Baerheim A, Fasmer O, Hjortdahl P, et al. Assesing medical students' attitudes towards learning communication skills-wich components of attitudes do we measure? BMC Medical Education 2007; 7: 4.

27. Liu O, Mao L, Frankel L, Xu J. Assessing critical thinking in higher education: The Heighten approach and preliminary validity evidence. Assessment \& Evaluation in Higher Education 2016; 41 (5): 677-94. 